\title{
Secretion of fibronectin by human pancreatic stellate cells promotes chemoresistance to gemcitabine in pancreatic cancer cells
}

\author{
Manoj Amrutkar ${ }^{1,2^{*}}$ (D), Monica Aasrum¹, Caroline S. Verbeke ${ }^{3,4}$ and Ivar P. Gladhaug ${ }^{2,5}$
}

\begin{abstract}
Background: Gemcitabine remains a cornerstone in chemotherapy of pancreatic ductal adenocarcinoma (PDAC) despite suboptimal clinical effects that are partly due to the development of chemoresistance. Pancreatic stellate cells (PSCs) of the tumor stroma are known to interact with pancreatic cancer cells (PCCS) and influence the progression of PDAC through a complex network of signaling molecules that involve extracellular matrix (ECM) proteins. To understand tumor-stroma interactions regulating chemosensitivity, the role of PSC-secreted fibronectin (FN) in the development of gemcitabine resistance in PDAC was examined.
\end{abstract}

Methods: PSC cultures obtained from ten different human PDAC tumors were co-cultured with PCC lines (AsPC-1, BxPC-3, Capan-2, HPAF-II, MIA PaCa-2, PANC-1 and SW-1990) either directly, or indirectly via incubation with PSCconditioned medium (PSC-CM). Gemcitabine dose response cytotoxicity was determined using MTT based cell viability assays. Protein expression was assessed by western blotting and immunofluorescence. PSC-CM secretome analysis was performed by proteomics-based LC-MS/MS, and FN content in PSC-CM was determined with ELISA. Radiolabeled gemcitabine was used to determine the capacity of PCCs to uptake the drug.

Results: In both direct and indirect co-culture, PSCs induced varying degrees of resistance to the cytotoxic effects of gemcitabine among all cancer cell lines examined. A variable degree of increased phosphorylation of ERK1/2 was observed across all PCC lines upon incubation with PSC-CM, while activation of AKT was not detected. Secretome analysis of PSC-CM identified 796 different proteins, including several ECM-related proteins such as FN and collagens. Soluble FN content in PSC-CM was detected in the range 175-350 ng/ml. Neither FN nor PSC-CM showed any effect on PCC uptake capacity of gemcitabine. PCCs grown on FN-coated surface displayed higher resistance to gemcitabine compared to cells grown on non-coated surface. Furthermore, a FN inhibitor, synthetic Arg-Gly-Asp-Ser (RGDS) peptide significantly inhibited PSC-CM-induced chemoresistance in PCCs via downregulation of ERK1/2 phosphorylation.

Conclusions: The findings of this study suggest that FN secreted by PSCs in the ECM plays a key role in the development of resistance to gemcitabine via activation of ERK1/2. FN-blocking agents added to gemcitabinebased chemotherapy might counteract chemoresistance in PDAC and provide better clinical outcomes.

Keywords: Pancreatic stellate cells, Gemcitabine, Fibronectin, Pancreatic cancer, Chemoresistance, Extracellular matrix

\footnotetext{
*Correspondence: manoj.amrutkar@medisin.uio.no

'Department of Pharmacology, Institute of Clinical Medicine, University of

Oslo, Blindern, 0316 Oslo, Norway

${ }^{2}$ Department of Hepato-Pancreato-Biliary Surgery, Institute of Clinical

Medicine, University of Oslo, PO Box 1171, Blindern, 0318 Oslo, Norway

Full list of author information is available at the end of the article
}

(c) The Author(s). 2019 Open Access This article is distributed under the terms of the Creative Commons Attribution 4.0 International License (http://creativecommons.org/licenses/by/4.0/), which permits unrestricted use, distribution, and reproduction in any medium, provided you give appropriate credit to the original author(s) and the source, provide a link to the Creative Commons license, and indicate if changes were made. The Creative Commons Public Domain Dedication waiver (http://creativecommons.org/publicdomain/zero/1.0/) applies to the data made available in this article, unless otherwise stated. 


\section{Background}

Pancreatic ductal adenocarcinoma (PDAC), generally referred to as pancreatic cancer, is a highly malignant disease characterized by late diagnosis, early metastasis, limited response to chemotherapy, and an extremely poor prognosis [1-3]. Despite significant advances in the understanding of the pathobiology of the disease over the past decades, overall 5-year survival rate has only marginally improved to merely 7\%, making it the fourth leading cause of cancerrelated mortality in the Western world [4]. Moreover, it is projected to rank second by 2030 [5]. While surgery offers the only potential curative treatment, only $15-20 \%$ of patients have resectable disease at the time of diagnosis, and these patients also carry the risk of disease recurrence within a year post-surgery [6]. Chemotherapy is therefore a crucial component in the treatment of the disease, both for unresectable (locally advanced or metastatic) disease or in the neoadjuvant and adjuvant settings [7]. Gemcitabine $(\mathrm{dFdC})$ is a nucleoside pyrimidine analogue that has long been the backbone of chemotherapy for PDAC in all stages of the disease $[8,9]$. However, gemcitabine has suboptimal clinical outcomes caused by molecular mechanisms limiting its cellular uptake, activation and overall efficacy, as well as by the development of chemoresistance within weeks of treatment initiation $[10,11]$.

The presence of a dense desmoplastic stroma that constitutes the bulk of the tumor mass is a prominent feature of PDAC $[12,13]$. The stroma is composed of several different cell types and various extracellular matrix (ECM) components such as collagens and fibronectin (FN), which provide structural integrity and initiate signaling cascades that promote tumor cell survival, proliferation, and migration [14-16]. The activated pancreatic stellate cell (PSC) - a type of cancer-associated fibroblast (CAF) - is the primary source of ECM components and also the major driver and organizer of the desmoplastic reaction in PDAC [17]. In the healthy pancreas, PSCs exist in a quiescent state, but in response to various pathophysiological stimuli such as injury, inflammation and induction of neoplasia in ductal epithelium, the PSCs are activated $[14,18]$. Through the secretion of excessive amounts of ECM components, activated PSCs induce the fibrosis that is characteristically found in PDAC [18, 19].

Several lines of evidence suggest that the tumor stroma contributes to gemcitabine chemoresistance through a range of mechanisms, including impairment of drug delivery due to decreased functional microvascularity, activation of anti-apoptotic pathways, promotion of epithelial-mesenchymal transition, and upregulation of tumor-supporting inflammatory cytokines [10, 2022 . While the cause of gemcitabine chemoresistance is clearly multifactorial and PSCs are implicated in several of these processes, the precise role of the PSCs in the development of chemoresistance is still not fully understood [23-26]. Previous studies have also indicated that pancreatic cancer cells (PCCs) embedded in collagen gels [27] or cultured on FN-coated dishes [28], show increased resistance to gemcitabine, suggesting the possibility that ECM-induced signals might be involved.

In the present study we hypothesized that secreted soluble factors implicated in gemcitabine chemoresistance in pancreatic cancer may be identified through analysis of the PSC secretome. To this end, we examined conditioned medium from ten primary human PSC cultures for its secretome content and evaluated its effects on PCC's gemcitabine chemosensitivity in order to explore the effects of ECM proteins on pancreatic cancer chemoresistance.

\section{Methods \\ Reagents}

Reagents were purchased from the following sources: Dulbecco's modified Eagle's medium containing $4.5 \mathrm{~g} / \mathrm{l}$ glucose (DMEM), penicillin-streptomycin (Pen-Strep), amphotericin B, Trypsin/EDTA (1X), fetal bovine serum (FBS) and Pierce ${ }^{\mathrm{TM}}$ BCA protein assay kit from Thermo Fisher Scientific (Waltham, MA, USA); bovine serum albumin (BSA), Collagen type I solution from rat tail, FN, gemcitabine hydrochloride, 3-(4,5-Dimethylthiazol-2-yl)2,5-Diphenyltetrazolium Bromide (MTT), phosphate buffered saline (PBS) and human fibronectin ELISA kit from Sigma-Aldrich (St Louis, MO, USA); FN inhibitor (RGDS) from Santa Cruz Biotechnology (Santa Cruz, CA, USA); $\left[{ }^{3} \mathrm{H}\right]$-gemcitabine from Moravek Biochemicals Inc. (Brea, CA, USA); Ultima Gold from Perkin Elmer (Waltham, MA, USA); PD98059 from Calbiochem (La Jolla, CA); human anti-alpha smooth muscle actin ( $\alpha$ SMA; BS66) from Nordic Biosite AB (Taby, Sweden); anti-AKT, anti-phospho-AKT, anti-glial fibrillary acidic protein (GFAP; GA5), anti-epithelial cell adhesion molecule (EpCAM; VU1D9), anti-p44/42 MAPK (ERK1/2), anti-phospho-ERK1/2, anti-vimentin (D21H3) and antiGAPDH from Cell Signaling Technology (Beverly, MA, USA); anti-phosphoinositol-3-kinases (PI3K) from Merck Life Science AS (Oslo, Norway); secondary HRPconjugated antibodies goat anti-mouse and goat antirabbit IgG from Bio-Rad Laboratories (Hercules, CA, USA); secondary Alexa Fluor-conjugated antibodies (anti-mouse and anti-rabbit) and DAPI from Jackson ImmunoResearch (West Grove, PA, USA).

\section{Cell isolation and culture}

Primary human PSCs obtained from tumor tissue sampled from surgical resection specimens with PDAC, were isolated and cultured by the outgrowth method as previously described [29, 30]. Cultures were established and propagated from ten different patients (designated as 


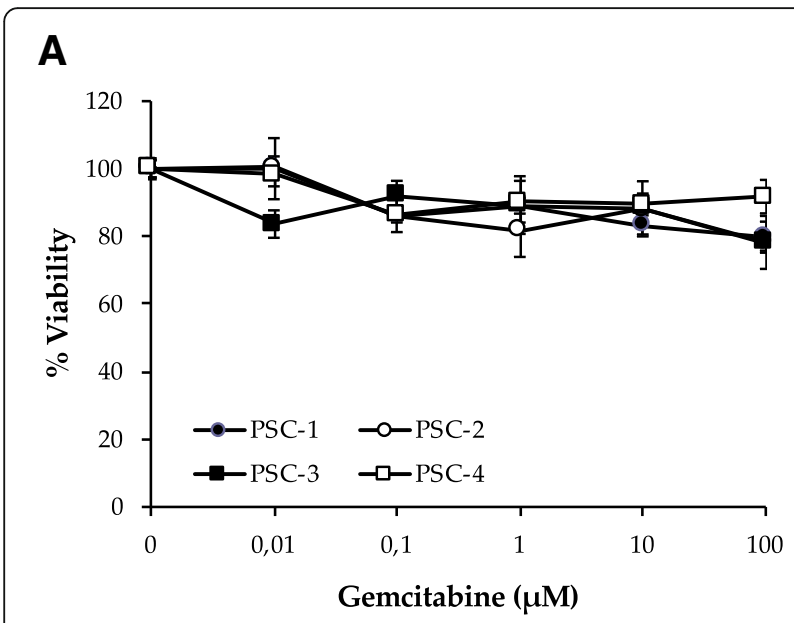

B

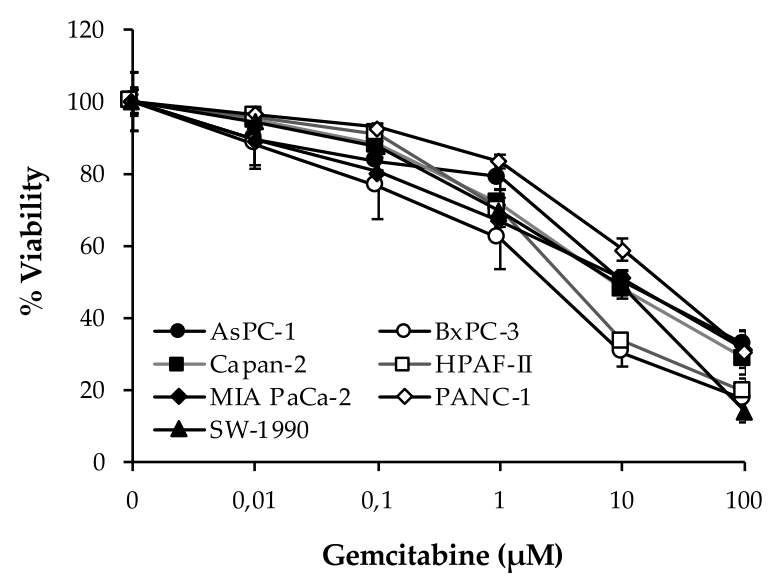

Fig. 1 PSCs are resistant to gemcitabine-induced cytotoxicity while gemcitabine dose response effects differ among various PCC lines. PDAC-derived human PSCs (a) and PCCs (b) seeded on 96-well plates at a density of 3000 cells/well, were incubated with increasing concentrations of gemcitabine for $48 \mathrm{~h}$ and evaluated for cell viability using the MTT assay. Data are mean \pm SEM of triplicate determinations. PCC, pancreatic cancer cell; PSC, pancreatic stellate cell

PSC-1 -2, - 3, - 4, - 5, -6, - 7, - 8, -9, and - 10). None of the ten patients received neoadjuvant therapy. Clinicopathological features of tumors are provided in Additional file 1: Table S1. Assessment of morphology and expression analysis of $\alpha \mathrm{SMA}$ and vimentin was performed to determine the purity of PSCs. PSC cultures between passage 3 and 8 were used for all experiments. The PSC cultures as well as the commercial PCC lines AsPC-1 (ATCC ${ }^{\ominus}$ CRL-1682 $^{\text {T从 }}$ ), BxPC-3 (ATCC ${ }^{\ominus}$ CRL$1687^{\mathrm{mm}}$ ), Capan-2 (ATCC ${ }^{\ominus}$ HTB- $^{\mathrm{m}} 0^{\mathrm{m}}$ ), HPAF-II $\left(\right.$ ATCC $^{\oplus}$ CRL-1997 ${ }^{\mathrm{rm}}$ ), MIA PaCa-2 (ATCC ${ }^{\oplus} \mathrm{CRL}^{-1420^{\mathrm{TM}}}$ ), PANC-1 $\left(\right.$ ATCC $^{\oplus}$ CRL-1469 $^{\text {tм }}$ ) and SW-1990 (ATCC ${ }^{\oplus}$ CRL-2172 $^{\text {TM }}$ ) obtained from American Type Culture Collection (ATCC, Manassas, VA, USA) were cultured and maintained in DMEM supplemented with 10\% FBS, 1\% PenStrep and 1\% amphotericin B and tested for mycoplasma contamination. None of the PCC lines are listed in the database of the International Cell Line Authentication Committee (ICLAC). For direct co-culture equal numbers of PSCs were seeded together with PCCs, while in indirect co-culture PCCs were incubated with PSCconditioned medium (PSC-CM), as described in Additional file 2: Figure S1.

\section{Immunocytochemistry}

Immunocytochemistry of PSCs was performed as described previously [31]. Briefly, PSCs cultured in 96-well plates were fixed in $4 \%$ formaldehyde, blocked in BSA and incubated overnight with primary antibodies against aSMA (1:50) and vimentin (1:200). The next day, cells were stained with Alexa Fluor-conjugated secondary antibodies (1:200) and DAPI was used for nuclear staining. Images were captured using EVOS FLoid Cell Imaging Station (Thermo Fisher Scientific).

\section{Preparation of conditioned medium}

The PSC-CM samples were prepared as described previously [31]. Briefly, sub-confluent PSC cultures were washed thoroughly with PBS and incubated for $48 \mathrm{~h}$ with fresh serum-free DMEM (SFM), conditioned medium was collected, centrifuged and stored at $-20^{\circ} \mathrm{C}$ until further use.

\section{Chemosensitivity}

Both PCCs and PSCs were cultured in 96-well plates at a density of 3000 cells/well and treated for $48 \mathrm{~h}$ with gemcitabine at a final concentration range of $0.01-$ $100 \mu \mathrm{M}$. The cell viability and $\mathrm{IC}_{50}$ values were determined using the MTT assay, in which the degree of formazan crystals formation is relative to the number of viable cells. $\mathrm{IC}_{50}$ values were determined by calculating the amount of gemcitabine required for inhibition of cell growth by $50 \%$ compared to untreated controls. Furthermore, PCCs were either cultured together with PSCs for $96 \mathrm{~h}$ (direct co-culture) or incubated with PSC-CM (indirect co-culture) for $24 \mathrm{~h}$, prior to treatment with gemcitabine for $48 \mathrm{~h}$ at a final concentration of $10 \mu \mathrm{M}$. The response to gemcitabine was evaluated with the MTT assay. The PSC-CM induced resistance to gemcitabine in various PCC lines was calculated using the following formula:

$$
\text { Drug resistance }(\%)=\frac{\text { \%viability in PSC-CM-\%viability in SFM }}{\text { \%viability in SFM }} 100
$$

\section{Secretome analysis}

Proteomics-based analysis of PSC-CM samples was performed as described previously with some modifications [31]. Briefly, the PSC-CM samples were concentrated down to $5 \%$ of the original volume using $10 \mathrm{kDa}$ cut off 
Table $1 \mathrm{I} \mathrm{C}_{50}$ values for gemcitabine in different PCC lines were calculated using GraphPad Prism software 6.0

\begin{tabular}{ll}
\hline Cell line & $I_{50}(\mu \mathrm{M})$ \\
\hline PANC-1 & 20.5 \\
AsPC-1 & 13.9 \\
Capan-2 & 9.7 \\
MIA PaCa-2 & 9.5 \\
SW-1990 & 6.2 \\
HPAF-II & 4.3 \\
BxPC-3 & 2.0 \\
\hline
\end{tabular}

Amicon Ultra centrifugal filter devices. Subsequently, the proteins were reduced, alkylated and digested overnight using trypsin (Promega). The next day, the peptides were desalted and concentrated before they were submitted to mass spectrometry (MS). Each peptide mixture was analyzed in three technical replicates by nEASY-LC coupled to QExactive Plus (ThermoElectron, Bremen, Germany) with EASY Spray PepMap ${ }^{\circ}$ RSLC column $(\mathrm{C} 18,2 \mu \mathrm{m}, 100 \AA, 75 \mu \mathrm{m} \times 50 \mathrm{~cm})$. Protein identification was performed using Proteome Discoverer 2.1 (Thermo Fisher Scientific) and Mascot 2.6 (MatrixScience, London, UK) search engine. The search criteria for Mascot searches were: trypsin digestion with two missed cleavage allowed, Carbamidomethyl (C) as fixed modification and Acetyl (N-term), Gln- > pyro-Glu (N-term Q), Oxidation (M) as dynamic modifications. The parent mass tolerance was $10 \mathrm{ppm}$ and MS/MS tolerance 0.1 Da. The SwissProt database for human entries supplemented with known contaminants provided by MaxQuant was used for the database searches. All of the reported protein identifications were statistically significant $(p<0.05)$ in Mascot and filtered in Proteome Discoverer for at least medium confidence identifications. The list of identified proteins were subjected to Kyoto Encyclopedia of Genes and Genomes (KEGG) database for pathway analysis [32] and Gene Ontology (GO) analysis was conducted using the DAVID Bioinformatics Database [33, 34]. The workflow of the procedure is illustrated in Additional file 3: Figure S2A.

\section{ELISA}

The amount of soluble FN present in the conditioned medium obtained from ten different PSC cultures was measured using human fibronectin ELISA kit, according to the manufacturer's protocol.

\section{Western blot analysis}

Total cell lysates were prepared using Laemmli buffer, and aliquots of protein were separated by electrophoresis (SDS-PAGE), as described previously [31]. The proteins were transferred to nitro-cellulose membranes, blocked in 5\% non-fat dry milk solution and incubated overnight with the primary antibodies as indicated. The next day, blots were washed and incubated with HRP-conjugated secondary antibodies at room temperature for $1 \mathrm{~h}$. The blots were visualized with LumiGLO ${ }^{\circ}$ (KPL, Gaithersburg, MD, USA) and their densitometric analysis was
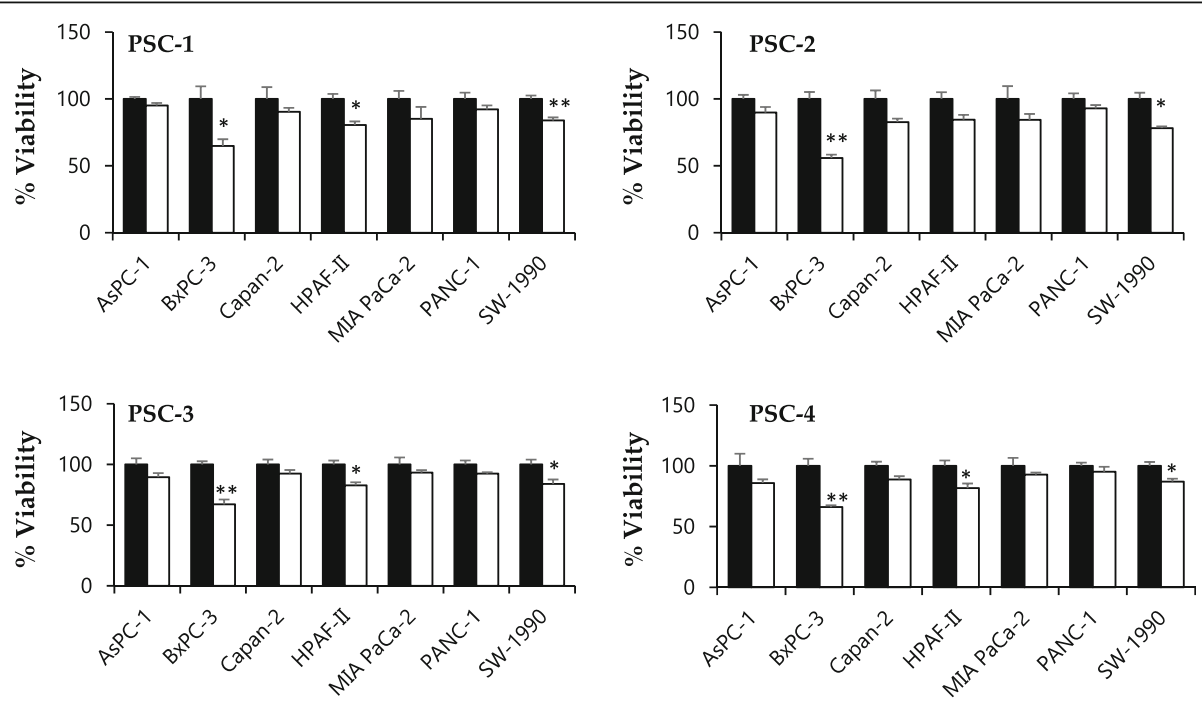

Control

Gemcitabine $(10 \mu \mathrm{M})$

Fig. 2 In direct co-culture with PSCS, PCCs develop resistance to gemcitabine. Equal numbers of PSCs and PCCs seeded together on 96-well plates, cultured for 4 days, were incubated with a single concentration of gemcitabine $(10 \mu \mathrm{M})$ for $48 \mathrm{~h}$ and evaluated for cell viability using MTT assay. Data are mean \pm SEM of triplicate determinations. ${ }^{*} p<0.05,{ }^{* *} p<0.01$ for control vs gemcitabine. PCC, pancreatic cancer cell; PSC, pancreatic stellate cell 


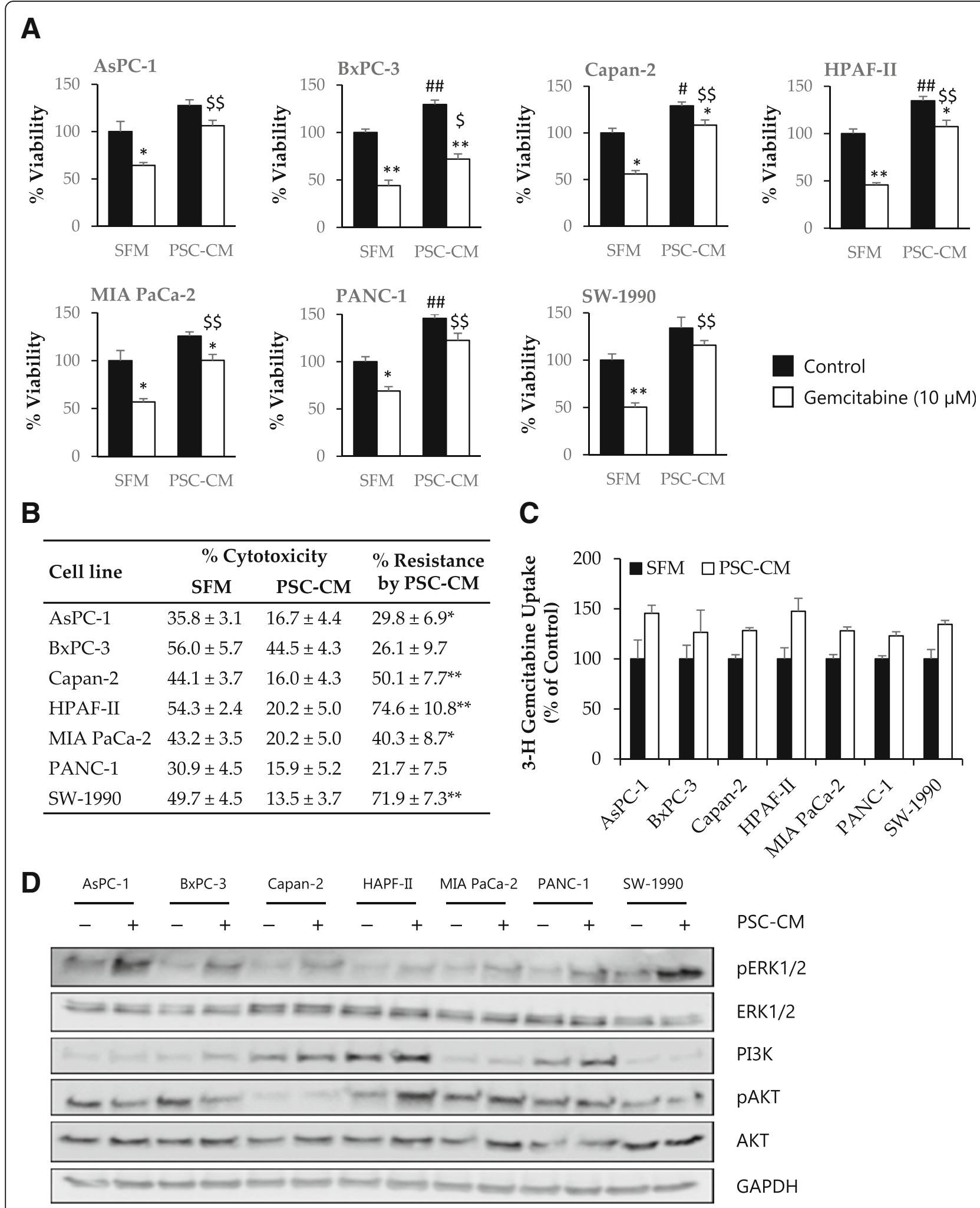

Fig. 3 (See legend on next page.) 
(See figure on previous page.)

Fig. 3 PSC-CM induces resistance to gemcitabine in PCCs but does not affect gemcitabine uptake. PCCs seeded on 96-well plates were incubated with SFM or PSC-CM for $24 \mathrm{~h}$ prior to incubation with $(\mathbf{a}, \mathbf{b})$ gemcitabine $(10 \mu \mathrm{M})$ for $48 \mathrm{~h}$ or with $(\mathbf{c})$ transport buffer containing $\left[^{3} \mathrm{H}\right]$ gemcitabine for $4 \mathrm{~h}$. For (a), cell viability was determined using the MTT assay. ${ }^{*} p<0.05$, ${ }^{* *} p<0.01$ for control vs gemcitabine; \#p $<0.05$, \#\#p $<$ 0.01 and $\$ p<0.05, \$ \$ p<0.01$ for SFM vs PSC-CM in control and gemcitabine groups, respectively. $\mathbf{b}$ The table indicates gemcitabine-induced cytotoxicity in percentage, and PSC-CM-induced resistance to gemcitabine, calculated by relative reduction in cytotoxicity between SFM and PSCCM. ${ }^{*} p<0.05,{ }^{* *} p<0.01$ comparing SFM with PSC-CM. For $\mathbf{c}$, gemcitabine uptake was determined by scintillation counting. Data are mean \pm SEM of triplicate determinations. $\mathbf{d}$ The PCCs were lysed and proteins subjected to immunoblotting using anti-pERK1/2, anti-ERK1/2, anti-PI3K, antipAKT, and anti-AKT antibodies. GAPDH was used as a loading control. PCC, pancreatic cancer cell; PSC, pancreatic stellate cell; PSC-CM, PSCconditioned medium; SFM, serum-free DMEM

performed using Labworks Software (UVP, Cambridge, UK).

\section{Gemcitabine uptake}

PCCs cultured to confluence in 96-well plates were washed with PBS and incubated with $100 \mu \mathrm{L}$ transport buffer (20 mM Tris, $3 \mathrm{mM} \mathrm{K} \mathrm{HPO}_{4}, 5 \mathrm{mM}$ glucose, 145 $\mathrm{mM} \mathrm{NaCl}, 1 \mathrm{mM} \mathrm{MgCl}$, and $1.2 \mathrm{mM} \mathrm{CaCl}_{2}$ ) containing $50 \mathrm{nM}\left[{ }^{3} \mathrm{H}\right]$-gemcitabine at $37^{\circ} \mathrm{C}$ for $4 \mathrm{~h}$. Subsequently, cells were lysed by incubation with $0.2 \mathrm{M} \mathrm{NaOH}$ for 15 min and lysates were then added to the scintillation tubes containing Ultima Gold. Cell-associated radioactivity in counts per minute (CPM) was determined using a liquid scintillation counter. Protein concentration of cell lysates was determined using BCA protein assay kit. $\left[{ }^{3} \mathrm{H}\right]$-gemcitabine transport was calculated by normalizing CPM to protein concentration for each well.

\section{Statistical analysis}

All values are expressed as a mean \pm standard error of mean (SEM). Statistical analysis of the results was performed using GraphPad Prism 6 (GraphPad Software), by an unpaired two-tailed Student's t test or by two-way ANOVA, followed by Tukey's post hoc test, with a value of $p<0.05$ considered statistically significant.

\section{Results}

Phenotypic characterization of the human primary PSC cultures

Expression of proteins considered characteristic of PSCs was analyzed by immunofluorescence and western blot analysis. All primary PSC cultures examined exhibited a fibroblast-like morphology that is characteristic of PSCs, and showed strong expression of the activation marker $\alpha \mathrm{SMA}$ and the mesenchymal marker vimentin, consistent with activated PSCs (Additional file 4: Figure S3A, B). GFAP was not detected in any of the PSC cultures (data not shown), consistent with the reported loss of expression during culturing [35]. None of the PSC cultures showed expression of the epithelial marker EpCAM (data not shown), excluding the possibility of contamination with epithelial cells during isolation.
Gemcitabine dose response chemosensitivity in PSCs and PCCs

MTT-based evaluation of dose response to gemcitabine in terms of cell viability in four PSC cultures revealed that these cells were resistant to the cytotoxic actions of gemcitabine. At a single concentration of gemcitabine $(10 \mu \mathrm{M})$ an average reduction of cell viability by merely $12 \%$ was observed (Fig. 1a). In contrast, gemcitabine differentially reduced the cell viability in the seven PCC lines studied (Fig. 1b). MTT assay revealed that gemcitabine dose dependently inhibited PCC viability and, at a single concentration of gemcitabine $(10 \mu \mathrm{M})$, the decrease in cell viability was 50, 70, 52, 66, 49, 41 and 51\% in AsPC-1, BxPC-3, Capan-2, HPAF-II, MIA PaCa-2, PANC-1 and SW-1990, respectively (Fig. 1b). Notably, a significant cell population in each PCC line was observed to be inherently resistant to gemcitabine, whereby the size of this subpopulation ranged from $14 \%$ of the total cell population in SW-1990 to $33 \%$ in AsPC-1 (Fig. 1b). The $\mathrm{IC}_{50}$ values suggested that PANC-1 $\left(\mathrm{IC}_{50}=\sim\right.$ $20 \mu \mathrm{M})$ and BxPC-3 $(\sim 2 \mu \mathrm{M})$ were least and most sensitive to gemcitabine, respectively (Table 1 ), which is in accordance with previous data [36].

\section{Effect of PSCs and their secretome on gemcitabine chemosensitivity of PCCs}

Each of the seven different PCCs were each co-cultured with four different PSC cultures for $96 \mathrm{~h}$ and evaluated for the response to gemcitabine at single concentration of $10 \mu \mathrm{M}$, using the MTT assay. Compared to the cell survival observed in PCCs alone (Fig. 1b), the cancer cells in co-cultures developed a considerable degree of resistance to gemcitabine, as indicated by a high cell survival than in monoculture (Fig. 2). For instance, coculture with PSC-1 reduced cell viability by a mere 9, 35, 9, 19, 15, 7 and $16 \%$ for AsPC-1, BxPC-3, Capan-2, HPAF-II, MIA PaCa-2, PANC-1 and SW-1990, respectively. Similar responses were observed for co-cultures with three further PSC culture (Fig. 2).

To evaluate the effect of PSCs-secreted soluble factors on cancer cell chemosensitivity, PCCs were incubated with PSC-CM for $24 \mathrm{~h}$ and analyzed for the response to the cytotoxic effects of gemcitabine using the MTT assay (Fig. 3a). All PCC lines studied developed a certain 
A Top pathways identified using KEGG pathway analysis

\begin{tabular}{llll}
\hline $\begin{array}{l}\text { Pathway } \\
\text { ID }\end{array}$ & Pathway Description & $\begin{array}{l}\text { Gene } \\
\text { Count }\end{array}$ & FDR \\
\hline 1100 & Metabolic pathways & 89 & $1.04 \mathrm{E}-08$ \\
4510 & Focal adhesion & 52 & $2.41 \mathrm{E}-26$ \\
4151 & PI3K-Akt signaling pathway & 51 & $3.89 \mathrm{E}-15$ \\
4512 & ECM-receptor interaction & 36 & $2.41 \mathrm{E}-26$ \\
5200 & Pathways in cancer & 33 & $5.03 \mathrm{E}-06$ \\
\hline
\end{tabular}

B

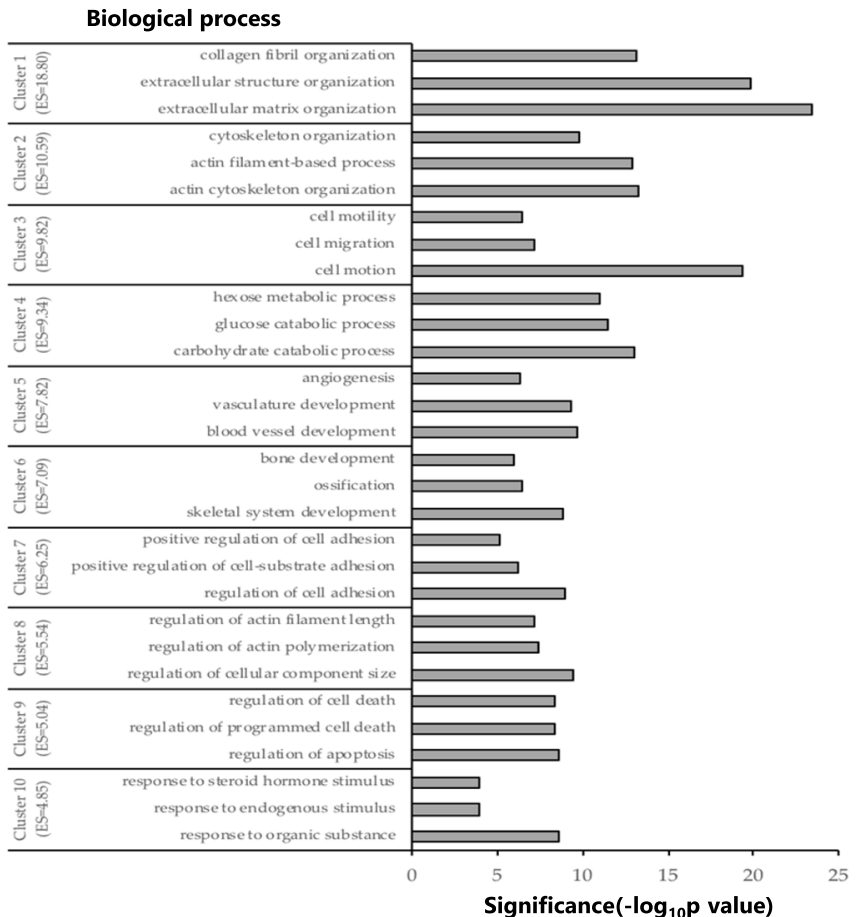

C

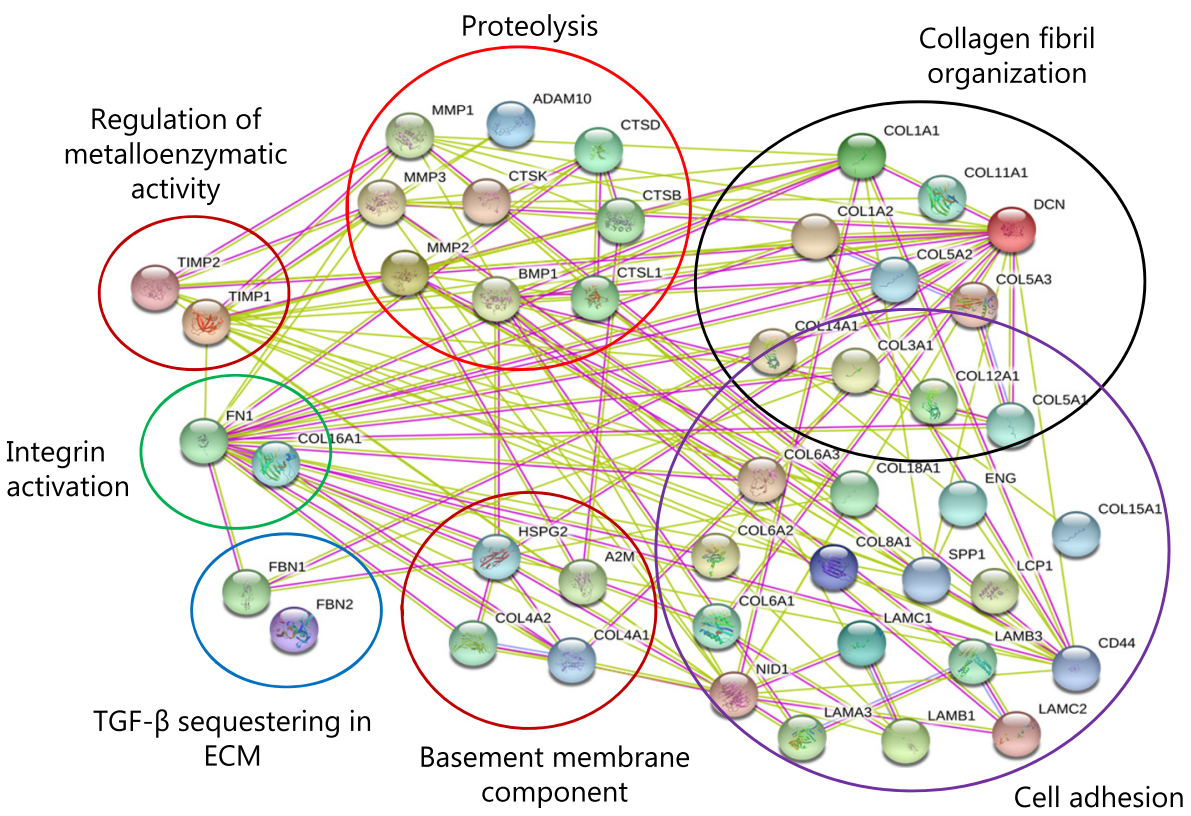

Fig. 4 (See legend on next page.) 
(See figure on previous page.)

Fig. 4 Proteome profiling of PSC-CM. Conditioned medium from ten different PSC cultures were subjected to proteomics analysis using LC-MS/ MS. a Top five KEGG pathways identified. $\mathbf{b}$ The proteins detected by LC-MS/MS were interrogated in terms of functional annotation by DAVID Bioinformatics Resource tool. The representative GO terms cluster groups with top 10 enrichment score are presented. The horizontal axis represents the significance ( $p$ value) for each term, while the vertical axis represents the GO categories for biological processes. c STRING network map of proteins involved in ECM remodeling and their categories based on molecular function. ECM, extracellular matrix; ES, enrichment score; FDR, false discovery rate; GO, gene ontology; KEGG, kyoto encyclopedia of genes and genomes; PSC, pancreatic stellate cell; STRING search tool for the retrieval of interacting genes/proteins

degree of resistance to gemcitabine, with the highest resistance being observed in HPAF-II ( 75\%). Although PANC-1 and BxPC-3 cells displayed a modest resistance (20-25\%), this change was not significant (Fig. 3a and b) . Notably, across the cell lines that were studied, coculture with PSC-CM had no significant effect on the uptake of radiolabeled gemcitabine in PCCs (Fig. 3c).

Of the many important signaling pathways that play a role in the pathobiology of PDAC, the ERK and PI3K/AKT signaling pathways are known to be also implicated in gemcitabine resistance [37, 38]. Substantiating the putative signaling effects of soluble factors in PSC-CM on the PCCs, a variable degree of increased phosphorylation of ERK1/2 was observed in all PCC lines upon incubation with PSC-CM (Fig. $3 \mathrm{~d}$ ), whereas activity of AKT or PI3K remained largely unaltered in the PCCs with the exception of HPAF-II, which displayed an increase in AKT phosphorylation (Fig. 3d).

\section{Secretome analysis of PSC-CM}

The composition of conditioned medium from various PSC cultures was investigated by proteomics-based analysis of the secretome preparations. A total of 796 different proteins (Homo sapiens) were identified, after the protein grouping performed by MaxQuant software. A complete list of all proteins together with their identification parameters are provided in Additional file 5: Table S2. The proteins with a number of peptides with high-medium confidence identified was between 200 and 600, across the ten PSC cultures studied (Additional file 3: Figure S2B). The identified proteins mainly belonged to the structural cellular components such as exosomes, extracellular vesicles and membrane-bound vesicles. Furthermore, to facilitate the interpretation of the results, a functional interaction network of the identified proteins was searched using the STRING software. The top five enriched KEGG pathway categories of the identified proteins are listed in Fig. 4a. To determine the functional role of the PSC-secreted proteins, a GO analysis was undertaken using the DAVID bioinformatic resource tool, which revealed a significant representation of biological process categories that were mainly related to ECM structure and organization, cytoskeleton organization, cell motility and adhesion, metabolism, regulation of cell death, and response to stimuli (Fig. 4b). A GO analysis also revealed a significant representation of molecular processes categories that were mainly related to carbohydrate and polysaccharide binding, enzyme activity, and calcium biding (Additional file 3: Figure S2C). GO functional annotation chart for KEGG analysis is presented in Additional file 6: Table S3. Furthermore, a total of 47 of all the identified proteins that are involved in ECM remodeling (Table 2), were grouped based on molecular functions as indicated (Fig. 4c).

\section{Effect of PSC-secreted FN on PCC chemoresistance}

In the PSC secretome, FN was detected with one of the highest number of peptides. The levels of soluble FN in conditioned medium from the ten PSCs cultures were in the range $175-350 \mathrm{ng} / \mathrm{ml}$ (Fig. 5a). Western blot analysis revealed that only the PSCs expressed FN, whereas all the PCC lines studied were negative (Fig. $5 b$ ), which is in accordance with previous findings [39]. Gemcitabine uptake capacity was not affected by FN-coating in any of the PCC lines studied (Fig. 5c). To evaluate the effect of FN on chemoresistance development, PCCs were cultured on FN-coated plates, and all cell lines with the exception of BxPC-3, displayed partial loss of the chemosensitivity to gemcitabine (Fig. $6 \mathrm{a}$ and b). However, the observed effect was considerably weaker than that of co-culture with PSC-CM (Fig. 3). To evaluate the effect of collagen - another major PSC-CM component - on chemoresistance development, PCCs were cultured on collagen-coated plates, which showed that none of the cell lines displayed reduction or loss of chemosensitivity to gemcitabine (Additional file 7: Figure S4). Furthermore, PCCs pre-incubated with PSC-CM and further incubated with RGDS, a FN inhibitor (H-ArgGly-Asp-Ser-OH), regained sensitivity to gemcitabine to some extent (Fig. 6a and b). Interestingly, in all PCC lines, treatment with RGDS also reversed the increase in phosphorylation of ERK1/2 to basal levels that had occurred during co-culture with PSC-CM (Fig. 6c). These findings were further confirmed by treatment with PD98059, a MEK/ERK inhibitor, which resulted in regained sensitivity to gemcitabine to an extent similar to that of RGDS (Additional file 8: Figure S5). 
Table $\mathbf{2}$ List of ECM remodeling proteins identified in PSC secretome

\begin{tabular}{|c|c|c|c|c|}
\hline Accession & Protein description & Gene symbol & Molecular weight [kDa] & Coverage \\
\hline P02751 & Fibronectin & FN1 & 262.5 & 66.7 \\
\hline P08123 & Collagen alpha-2(I) chain & COL1A2 & 129.2 & 82.3 \\
\hline P02452 & Collagen alpha-1(I) chain & COL1A1 & 138.9 & 81.1 \\
\hline P02461 & Collagen alpha-1(III) chain & COL3A1 & 138.5 & 55.3 \\
\hline P12109 & Collagen alpha-1(VI) chain & COL6A1 & 108.5 & 61.1 \\
\hline P35555 & Fibrillin-1 & FBN1 & 312.0 & 44.7 \\
\hline P12111 & Collagen alpha-3(VI) chain & COL6A3 & 343.5 & 48.1 \\
\hline P08253 & $72 \mathrm{kDa}$ type IV collagenase & MMP2 & 73.8 & 73.5 \\
\hline Q99715 & Collagen alpha-1(XII) chain & COL12A1 & 332.9 & 35.0 \\
\hline P11047 & Laminin subunit gamma-1 & LAMC1 & 177.5 & 38.1 \\
\hline P98160 & Heparan sulfate proteoglycan core protein & HSPG2 & 468.5 & 26.3 \\
\hline P08572 & Collagen alpha-2(IV) chain & COL4A2 & 167.4 & 41.1 \\
\hline P03956 & Interstitial collagenase & MMP1 & 54.0 & 65.9 \\
\hline P05997 & Collagen alpha-2( $\mathrm{V})$ chain & COL5A2 & 144.8 & 50.9 \\
\hline P01033 & Metalloproteinase inhibitor 1 & TIMP1 & 23.2 & 58.9 \\
\hline P20908 & Collagen alpha- $1(V)$ chain & COL5A1 & 183.4 & 21.0 \\
\hline P07585 & Decorin & DCN & 39.7 & 54.3 \\
\hline P12110 & Collagen alpha-2(VI) chain & COL6A2 & 108.5 & 44.2 \\
\hline P16035 & Metalloproteinase inhibitor 2 & TIMP2 & 24.4 & 67.7 \\
\hline P07942 & Laminin subunit beta-1 & LAMB1 & 197.9 & 32.7 \\
\hline P02462 & Collagen alpha-1(IV) chain & COL4A1 & 160.5 & 15.5 \\
\hline P14543 & Nidogen-1 & NID1 & 136.3 & 23.4 \\
\hline P12107 & Collagen alpha-1(XI) chain & COL11A1 & 181.0 & 14.2 \\
\hline P01023 & Alpha-2-macroglobulin & $\mathrm{A} 2 \mathrm{M}$ & 163.2 & 33.2 \\
\hline P07858 & Cathepsin B & CTSB & 37.8 & 46.3 \\
\hline P13497 & Bone morphogenetic protein 1 & BMP1 & 111.2 & 26.7 \\
\hline P16112 & Aggrecan core protein & ACAN & 250.0 & 10.2 \\
\hline P16070 & CD44 antigen & CD44 & 81.5 & 6.3 \\
\hline Q13443 & Disintegrin and metalloproteinase domain-containing protein 9 & ADAM9 & 90.5 & 5.5 \\
\hline P13796 & Plastin-2 & LCP1 & 70.2 & 12.8 \\
\hline Q07092 & Collagen alpha-1(XVI) chain & COL16A1 & 157.7 & 5.9 \\
\hline P27658 & Collagen alpha-1(VIII) chain & COL8A1 & 73.3 & 18.1 \\
\hline P07339 & Cathepsin D & CTSD & 44.5 & 38.3 \\
\hline P08254 & Stromelysin-1 & MMP3 & 53.9 & 24.1 \\
\hline P35556 & Fibrillin-2 & FBN2 & 314.6 & 4.1 \\
\hline Q05707 & Collagen alpha-1 (XIV) chain & COL14A1 & 193.4 & 9.4 \\
\hline Q13751 & Laminin subunit beta-3 & LAMB3 & 129.5 & 8.7 \\
\hline Q13753 & Laminin subunit gamma-2 & LAMC2 & 130.9 & 8.5 \\
\hline 014672 & Disintegrin and metalloproteinase domain-containing protein 10 & ADAM10 & 84.1 & 15.1 \\
\hline P07711 & Cathepsin L1 & CTSL1 & 37.5 & 20.4 \\
\hline P10451 & Osteopontin & SPP1 & 35.4 & 19.1 \\
\hline P17813 & Endoglin & ENG & 70.5 & 8.2 \\
\hline Q16787 & Laminin subunit alpha-3 & LAMA3 & 366.4 & 2.8 \\
\hline P39059 & Collagen alpha-1(XV) chain & COL15A1 & 141.6 & 6.2 \\
\hline
\end{tabular}


Table 2 List of ECM remodeling proteins identified in PSC secretome (Continued)

\begin{tabular}{lllll}
\hline Accession & Protein description & Gene symbol & Molecular weight [kDa] & Coverage \\
\hline P39060 & Collagen alpha-1(XVIII) chain & COL18A1 & 178.1 & 5.2 \\
P25940 & Collagen alpha-3(V) chain & COL5A3 & 172.0 & 2.7 \\
P43235 & Cathepsin K & CTSK & 36.9 & 5.5 \\
\hline
\end{tabular}

\section{Discussion}

Crosstalk between mesenchymal stromal cells and epithelial cancer cells has recently been implicated in the development of chemoresistance in pancreatic cancer. In particular, increasing evidence suggest that PSCs in the tumor stroma are both direct and indirect drivers of pancreatic cancer chemoresistance [23]. Since the 1997 report by Burris et al. [8], gemcitabine has remained a cornerstone of pancreatic cancer chemotherapy despite only marginal effects on patient survival, to a large extent caused by the high intrinsic (de novo) and acquired (therapy-induced) chemoresistance [40]. Thus, knowledge about the underlying mechanisms of drug resistance in PDAC is critical in attempts to develop new and effective treatment regimens for this lethal condition.

In the present study we performed a proteomics-based analysis of the PSC secretome to identify PSC-secreted factors that possibly affect PCCs in signaling pathways involved in the development of resistance to gemcitabine.
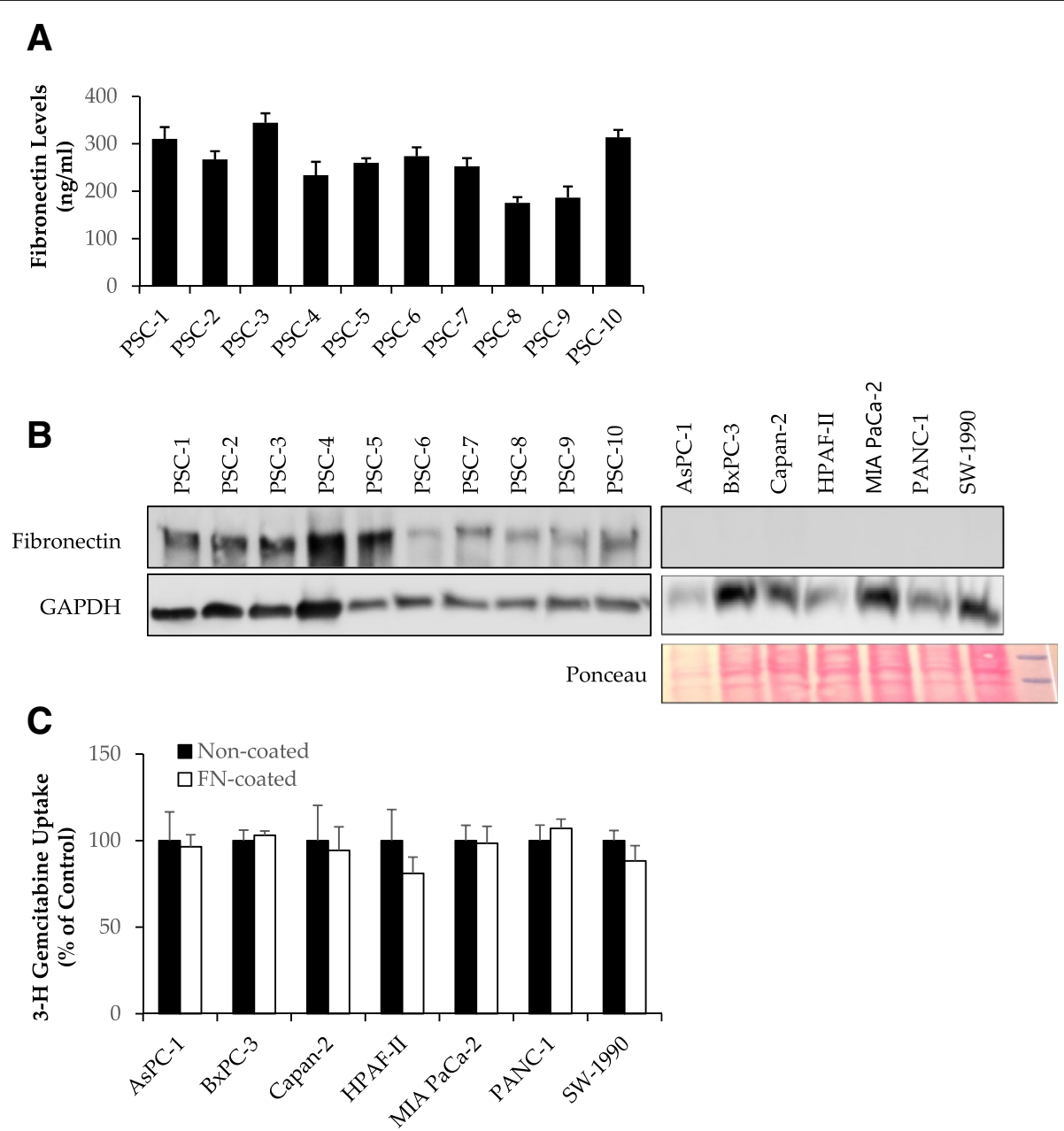

Fig. 5 Measurement of FN content in PSC-CM and its effect on gemcitabine uptake by PCCs. a Amount of soluble FN in conditioned medium from ten different PSC cultures was measured by ELISA. b Both PCCS and PSCS were lysed and proteins subjected to immunoblotting using antiFN antibody. GAPDH was used as a loading control. For PCCs, ponceau image also indicates loading. c Cancer cells seeded on 96-well plates with- or without FN-coating were incubated for $24 \mathrm{~h}$ prior to incubation with transport buffer containing $\left.{ }^{3} \mathrm{H}\right]$-gemcitabine for $4 \mathrm{~h}$. Data are mean \pm SEM of triplicate determinations. FN, fibronectin; PCC, pancreatic cancer cell; PSC, pancreatic stellate cell 
A
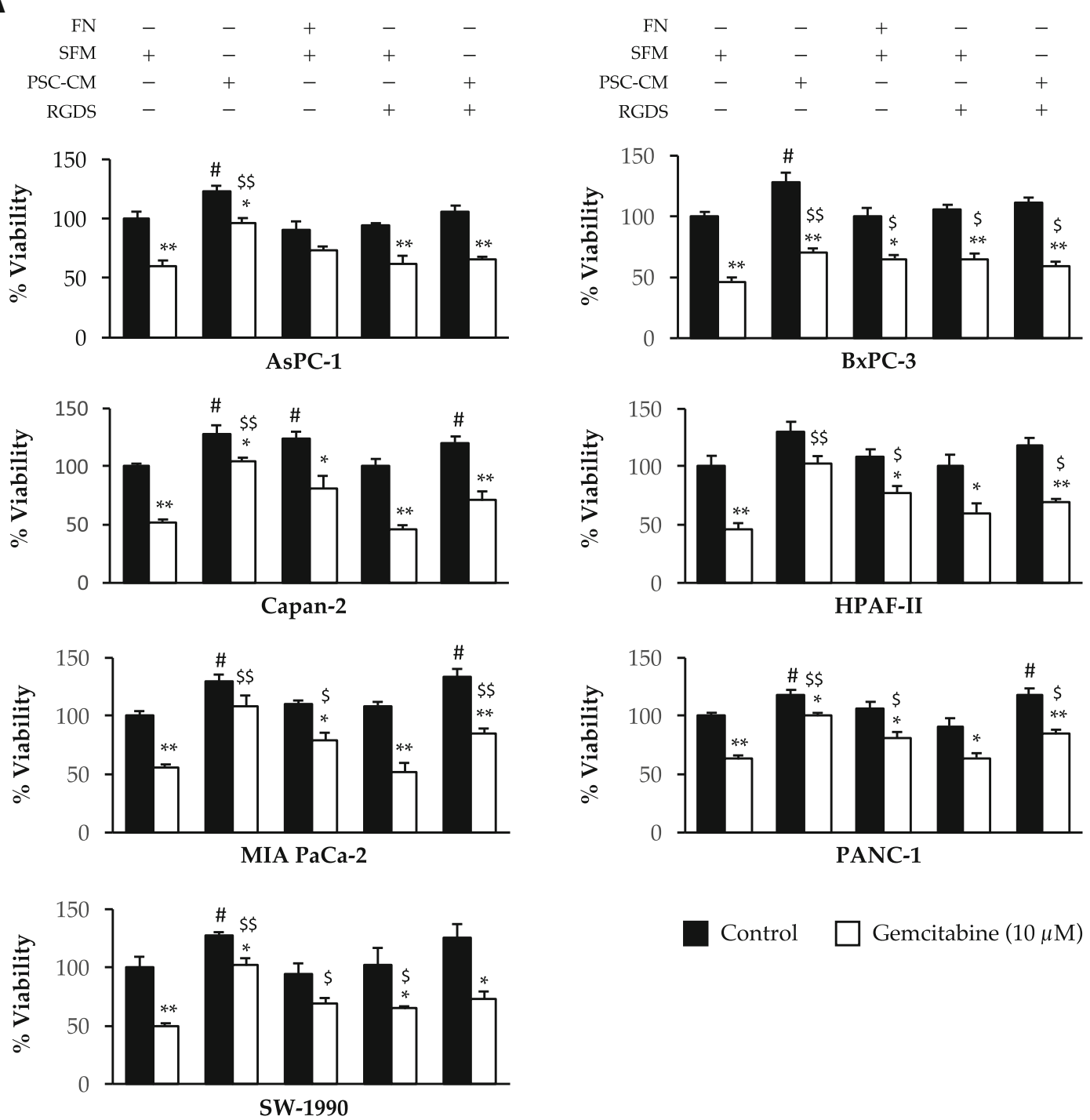

Control $\square$ Gemcitabine $(10 \mu \mathrm{M})$

B

\begin{tabular}{llllll}
\hline \multirow{2}{*}{ Cell lines } & \multicolumn{4}{c}{ \% Cytotoxicity } & \multicolumn{2}{c}{$\%$ Resistance } \\
& SFM & PSC-CM & PSC-CM & PSC-CM & PSC-CM \\
+ RGDS & RGDS \\
\hline AsPC-1 & $39.7 \pm 4.6$ & $20.6 \pm 2.8$ & $39.4 \pm 4.1$ & $31.7 \pm 4.6^{*}$ & $4.0 \pm 3.4$ \\
BxPC-3 & $53.2 \pm 3.1$ & $45.2 \pm 2.9$ & $46.3 \pm 2.6$ & $17.1 \pm 6.3$ & $14.8 \pm 5.6$ \\
Capan-2 & $48.4 \pm 2.9$ & $18.3 \pm 2.4$ & $39.1 \pm 4.7$ & $58.3 \pm 4.6^{* *}$ & $17.9 \pm 9.1$ \\
HPAF-II & $53.5 \pm 5.0$ & $21.4 \pm 5.4$ & $40.9 \pm 1.8$ & $69.0 \pm 11.7^{*}$ & $27.0 \pm 3.8$ \\
MIA PaCa-2 & $44.2 \pm 2.6$ & $16.7 \pm 7.7$ & $42.0 \pm 2.7$ & $49.2 \pm 13.8^{*}$ & $13.1 \pm 6.2$ \\
PANC-1 & $37.2 \pm 3.4$ & $15.4 \pm 2.4$ & $54.8 \pm 1.1$ & $34.5 \pm 3.8^{* *}$ & $12.9 \pm 5.2$ \\
SW-1990 & $50.7 \pm 2.8$ & $20.2 \pm 5.0$ & $47.0 \pm 7.4$ & $62.0 \pm 10.2^{* *}$ & $18.0 \pm 9.9$ \\
\hline
\end{tabular}

C

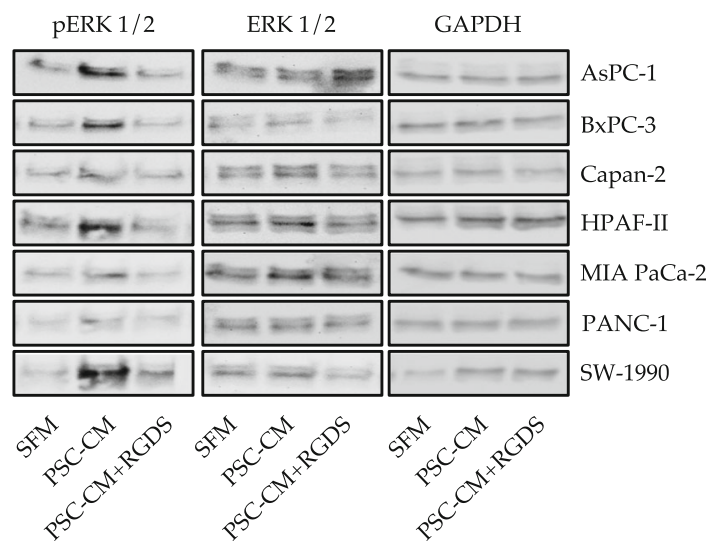

Fig. 6 (See legend on next page.) 
(See figure on previous page.)

Fig. 6 FN-inhibitor blocks chemoresistance inducing effect of PSC-secreted FN on PCCs via downregulation of ERK1/2 phosphorylation. a PCCs seeded on 96-well plates with- or without FN-coating as indicated. Cell were incubated with SFM or PSC-CM for $24 \mathrm{~h}$ and/or RGDS (FN inhibitor; $20 \mu \mathrm{M})$ for $4 \mathrm{~h}$ prior to incubation with gemcitabine $(10 \mu \mathrm{M})$ for $48 \mathrm{~h}$. Cell viability was determined using the MTT assay. ${ }^{*} p<0.05$, ${ }^{* *} p<0.01$ for control vs gemcitabine; $\# p<0.05$, \#\#p $<0.01$ and $\$ p<0.05$, $\$ p<0.01$ for SFM vS PSC-CM/FN/RGDS in control (black bars) and gemcitabine (white bars), respectively. $\mathbf{b}$ The table indicates gemcitabine-induced cytotoxicity in percentage, PSC-CM-induced resistance to gemcitabine, and resistance following subsequent incubation with RGDS. The resistance developed was calculated by relative reduction in cytotoxicity between SFM and PSC-CM or PSC-CM + RGDS. ${ }^{*} p<0.05,{ }^{* *} p<0.01$ comparing SFM with PSC-CM or SFM vs PSC-CM + RGDS. $\mathbf{c}$ The cells were lysed and proteins subjected to immunoblotting using anti-pERK1/2 and anti-ERK1/2 antibodies. GAPDH was used as a loading control. Data are the mean \pm SEM of triplicate determinations. FN, fibronectin; PCC, pancreatic cancer cell; PSC, pancreatic stellate cell; PSC-CM, PSC-conditioned medium; SFM, serum-free DMEM

The analysis resulted in identification of 796 proteins, the majority of which were ECM related proteins including FN and collagens. When cultured on FN-coated surface, all PCC lines tested displayed to a varying degree an increased resistance to gemcitabine, suggesting that ECM proteins are implicated in resistance-induction, in accordance with a previous report by Miyamoto et al. [28]. We observed that both in indirect and direct co-culture, PSCs induced a varying degree of gemcitabine resistance in all PCC lines tested. Notably, a synthetic FN inhibitor (RGDS peptide, which blocks integrin receptor function) counteracted to a limited extent resistance-induction by the PSCs, suggesting that PSC-secreted FN plays a key role in the development of gemcitabine resistance. However, the effect of PSC-CM on the development of chemoresistance was significantly higher compared to the effect of FN alone, which suggests that secretome components other than FN may also contribute to chemoresistance to gemcitabine.

Several tumor-modifying interactions between PSCs and PCCs have been reported in the literature, including effects of PSCs on cancer cell proliferation, migration and invasion as well as resistance to chemotherapeutic agents [10, 35, 41-44]. The panel of PCCs examined in the present study differ in their sensitivity to gemcitabine, as the $\mathrm{IC}_{50}$ for gemcitabine was approximately ten times higher in the relatively resistant PANC-1 cells compared to the most sensitive BxPC-3 cells. Upon direct co-culture with the tumor-derived PSCs, all the PCC lines examined became relatively resistant, albeit to a variable degree, indicating possible cell-line specific effects. In this context it should be recognized that the cancer cells differ in their mutation status; notably the BxPC-3 cell line is the only one with wild type KRAS [45]. Although several mechanisms could conceivably account for the observed loss of sensitivity to gemcitabine during direct co-culture, effects of PSC-secreted components on cancer cells seems an obvious possibility. This was substantiated with the findings from indirect co-culture experiments in which incubation of PCCs with PSC-CM resulted in the development of varying degrees of resistance to gemcitabine across all cell lines examined. Notably, direct co-culture experiments also suggest that, despite the differences in origin (tumor biopsies from different patients), PSCs modulated the chemosensitivity to gemcitabine of neighboring cancer cells to a similar extent.

Shortcomings of PDAC chemotherapy have been ascribed to poor drug availability as well as stromal factors with the ability to induce chemoresistance. Intracellular uptake of gemcitabine requires nucleoside transporters hENTs and hCNTs, and their expression correlate with gemcitabine sensitivity and overall survival among gemcitabine-treated PDAC patients. Loss of nucleoside transporters leads to development of resistance to gemcitabine [10, 39, 46, 47]. In the present study, neither

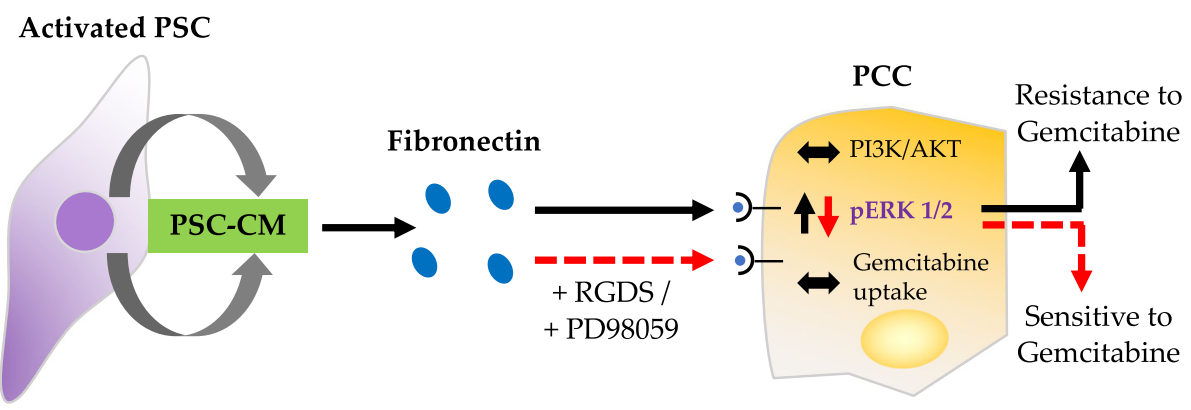

Fig. 7 PSC-secreted fibronectin promotes chemoresistance to gemcitabine in PCCS. PSC-secreted fibronectin induces resistance to gemcitabine in PCCs via upregulation of ERK1/2 phosphorylation, while no change in gemcitabine uptake. Addition of RGDS (fibronectin receptor blocking agent) or PD98059 (MEK/ERK inhibitor) to PCCs incubated with PSC-CM protect from chemoresistance progression, via inhibition of ERK1/2 phosphorylation. PCC, pancreatic cancer cell; PSC, pancreatic stellate cell; PSC-CM, PSC-conditioned medium 
PSC-CM nor FN had any significant effect on the overall gemcitabine uptake, suggesting that the observed loss of chemosensitivity may not be an effect of PSCs on the gemcitabine uptake capacity of PCCs. Furthermore, KEGG pathways analysis of the PSC secretome indicated that PSC secretion of FN could be a major determinant for induction of gemcitabine chemoresistance. Indeed, FN is a major ECM component that is primarily secreted by fibroblasts and cancer-associated fibroblasts [48, 49], and most of the biological effects of $\mathrm{FN}$ in as diverse processes as embryogenesis, wound healing and tissue homeostasis $[48,50-53]$ are mediated through integrins. The latter are a family of heterodimeric and transmembrane receptors that lead to activation of several signal transduction pathways, including ERK, p38 and PI3K/ AKT [54, 55].

Both the extracellular signal-regulated kinase (ERK) pathway and the PI3K/AKT pathway are known to be important in the pathobiology of PDAC $[56,57]$ and also implicated in gemcitabine chemoresistance [37, 38]. High ERK1/2 activity has also been reported to contribute to the gemcitabine resistance in pancreatic cancer, by protecting tumor cells from chemotherapy-induced apoptosis $[58,59]$. In this study, incubation of PCCs with PSC-CM-induced an increase in phosphorylation of ERK $1 / 2$, and this effect was counteracted by the synthetic FN inhibitor, suggesting that of many possible signaling pathways, PSCs mediate the resistance to gemcitabine via ERK1/2 activity. This was further confirmed by treatment with PD98059, a MEK/ERK inhibitor, which resulted in regained chemosensitivity for gemcitabine in PCCs. Notably, FN displayed profound inhibitory effects on ERK signaling whereas the gemcitabine sensitivity was not inhibited to the same extent, suggesting that other mechanisms or signaling pathways also might be involved. The PI3K/AKT pathway has been reported to be linked to anti-apoptotic signal transduction and chemoresistance in the PCC lines PK1 and PK8 as well as in an orthotopic xenograft model from the same cells $[60,61]$. However, it was later reported by Arlt et al. [62], that no such role of PI3K/AKT was found in a study employing BxPc-3, Capan-1 and PancTu-1 cells. In the present study, no change in activity of AKT or PI3K was observed in response to PSC$\mathrm{CM}$ across the seven different PCCs examined. A previous study by Sawai et al. [63] showed that the basement membrane proteins FN and collagen IV induced invasion of pancreatic cancer cells via increased ERK1/2 phosphorylation and that this effect was inhibited by RGDS peptide and $\beta 1$-integrin antibody as well as by the MEK/ERK inhibitor PD98059. Thus, the results of the present study are consistent with the notion that fibronectin-integrin mediated ERK signaling is involved in pancreatic cancer cell proliferation and survival.
In addition to $\mathrm{FN}$, other matrix components, particularly collagens, may contribute to stroma-induced chemoresistance [64]. High levels of expression of collagen genes were associated with drug resistance in ovarian and breast cancer cell lines [65, 66]. Knowledge regarding the role of different collagens in chemoresistance in pancreatic cancer is very limited. PANC-1 cells cultured in $3 \mathrm{D}$ collagen I have been reported to show increased gemcitabine resistance due to increased histone acetylation, also possibly affecting gene expression through activation of PI3K/AKT and ERK signaling pathways, leading to increased proliferation despite drug treatment $[27,67]$.

Despite recent advances in combination therapies, gemcitabine remains a cornerstone of neo-adjuvant, adjuvant, and palliative therapy for advanced PDAC [68]. A better understanding of the causes of gemcitabine chemoresistance, including the role of the tumor stroma, is critical to the development of novel treatment strategies. It seems obvious that developing new therapies for PDAC is a much more complex challenge than merely targeting the cancer cells alone. Recent studies in genetically modified mouse models and clinical trials targeting PSCs have shown conflicting results, with both positive and negative effects on cancer progression and responses to gemcitabine [69]. Understanding the aspects of PSC biology that promote therapy resistance and identifying the relevant signaling mechanisms are important steps in the development of effective stroma-targeting treatment strategies.

\section{Conclusions}

In conclusion, the findings of the present study suggest that PSC-secreted FN promotes high ERK1/2 activity in cancer cells and thereby protects the PCCs from gemcitabine-induced cytotoxicity (Fig. 7). Combined use of an FN inhibitor and gemcitabine might result in reduced chemoresistance to gemcitabine. Further studies are needed to elucidate the mechanisms by which FN blocking agents added to gemcitabine-based chemotherapy might counteract chemoresistance in PDAC thereby providing better clinical outcomes.

\section{Additional files}

Additional file 1: Table S1. Clinicopathological features of pancreatic adenocarcinoma (PADC) tumors. (DOCX $14 \mathrm{~kb}$ )

Additional file 2: Figure S1. Experimental set-up for co-culture experiments. Monolayer of PCCS (A), PSCs (B), direct co-culture of PCCs with PSCs (C) and indirect co-culture of PSCs with PCCS (D). PCC, pancreatic cancer cell; PSC, pancreatic stellate cell. (PPTX $61 \mathrm{~kb}$ )

Additional file 3: Figure S2. Secretome analysis of PSC-conditioned medium. (A) Flow-chart describing procedure for conditioned medium collection, processing and proteome analysis of the PSC secretome. (B) Conditioned medium collected from ten different PSC cultures were 
subjected to proteomic analysis by LC-MS/MS. Graph indicates the number of proteins identified with high confidence from each sample. (C) The proteins detected by LC-MS/MS were interrogated in terms of functional annotation by DAVID Bioinformatics Resource tool. The representative GO terms cluster groups with top 10 enrichment score are presented. The horizontal axis represents the significance ( $p$ value) for each term, while the vertical axis represents the $\mathrm{GO}$ categories for biological processes. GO, gene ontology; KEGG, kyoto encyclopedia of genes and genomes; PSC, pancreatic stellate cell; PSC-CM, PSC-conditioned medium; SFM, serum-free DMEM; STRING, search tool for the retrieval of interacting genes/proteins. (PPTX $3662 \mathrm{~kb}$ )

Additional file 4: Figure S3. Characterization of human PSCs. (A) PSCS immunostained with anti-aSMA (green) and anti-vimentin (red) antibodies. Nuclei stained with DAPI (blue). Scale bar $=100 \mu \mathrm{M}$. (B) The cells were lysed and proteins subjected to immunoblotting using anti-aSMA and anti-vimentin antibodies. GAPDH was used as a loading control. PSC, pancreatic stellate cell; aSMA, a-smooth muscle actin. (PPTX $4411 \mathrm{~kb}$ )

Additional file 5: Table S2. A complete list of all PSC secretome proteins together with their identification parameters. (XLSX 154 kb) Additional file 6: Table S3. Gene ontology functional annotation. (XLSX $15 \mathrm{~kb}$ )

Additional file 7: Figure S4. Effect of collagen on gemcitabine sensitivity. PCCs seeded on 96-well plates with- or without collagencoating as indicated. Cells were incubated with SFM for $24 \mathrm{~h}$ prior to incubation with gemcitabine $(10 \mu \mathrm{M})$ for $48 \mathrm{~h}$. Cell viability was determined using the MTT assay. Data are the mean \pm SEM of triplicate determinations. ${ }^{*} p<0.05,{ }^{*} p<0.01$ for control vs gemcitabine; $\# p<0.05$, for SFM vs collagen in control and gemcitabine groups. SFM, serum-free DMEM. (PDF $41 \mathrm{~kb}$ )

Additional file 8: Figure S5. Both FN-inhibitor (RGDS) and ERK-inhibitor (PD98059) block PSC-CM induced chemoresistance to gemcitabine. PCCs seeded on 96-well plates were incubated with SFM or PSC-CM for $24 \mathrm{~h}$ and/or RGDS $(20 \mu \mathrm{M})$ or PD98059 $(20 \mu \mathrm{M})$ for $4 \mathrm{~h}$ prior to incubation with gemcitabine $(10 \mu \mathrm{M})$ for $48 \mathrm{~h}$. Cell viability was determined using the MTT assay. Data are the mean \pm SEM of triplicate determinations. ${ }^{*} p<0.05$, ${ }^{* *} p<0.01$ for control vs gemcitabine; $\# p<0.05$, \#\#p $<0.01$ and $\$ p<0.05$, $\$ \$ p<0.01$ for SFM vs PSC-CM/RGDS/ PD98059 in control and gemcitabine, respectively. FN, fibronectin; PSC, pancreatic stellate cell; PSC-CM, PSC-conditioned medium; SFM, serum-free DMEM. (PDF 48 kb)

\section{Abbreviations}

CAFs: Cancer-associated fibroblasts; ECM: Extracellular matrix; FN: Fibronectin; PCC: Pancreatic cancer cell; PDAC: Pancreatic ductal adenocarcinoma; PSC: Pancreatic stellate cell; PSC-CM: PSC-conditioned medium; SFM: Serumfree DMEM

\section{Acknowledgements}

Mass spectrometry (secretome) analysis was performed at the Proteomics Core Facility, Department of Immunology, University of Oslo and Oslo University hospital, Norway.

\section{Authors' contributions}

MAmrutkar and IPG designed the project, analyzed the data and wrote the manuscript. MAmrutkar and MAasrum performed the experiments. CSV was responsible for sampling, logistics and pathological evaluation of human pancreatic cancer tissue specimens. IPG supervised the project. All authors commented on and approved of the submitted manuscript.

\section{Funding}

The authors acknowledge the financial support provided by the University of Oslo, Norway and a grant from the South-Eastern Norway Regional Health Authority (Helse Sør-Øst, grant no. 2016090), for running costs of the project. The funders had no role in the study design, data collection, data analysis and interpretation, or manuscript writing nor in the decision to submit the manuscript for publication

\section{Availability of data and materials}

The datasets used and/or analyzed during the current study are available from the corresponding author on reasonable requests.

\section{Ethics approval and consent to participate}

The study protocol and patient consent documents were approved by the Regional Committee for Medical and Health Research Ethics (REC South East, project number 2015/738) and followed the Helsinki Declaration. Written informed consent was obtained from all study participants, whose tumor tissue was used for the study. Commercial cancer cell lines were purchased from ATCC, and did not require ethics approval for their use in this study.

\section{Consent for publication}

Not applicable.

\section{Competing interests}

The authors declare that they have no competing interests.

\section{Author details}

'Department of Pharmacology, Institute of Clinical Medicine, University of Oslo, Blindern, 0316 Oslo, Norway. ${ }^{2}$ Department of Hepato-Pancreato-Biliary Surgery, Institute of Clinical Medicine, University of Oslo, PO Box 1171, Blindern, 0318 Oslo, Norway. ${ }^{3}$ Department of Pathology, Institute of Clinical Medicine, University of Oslo, Blindern, 0316 Oslo, Norway. ${ }^{4}$ Department of Pathology, Oslo University Hospital Rikshospitalet, Nydalen, 0424 Oslo, Norway. ${ }^{5}$ Department of Hepato-Pancreato-Biliary Surgery, Oslo University Hospital Rikshospitalet, Nydalen, 0424 Oslo, Norway.

\section{Received: 18 December 2018 Accepted: 6 June 2019}

Published online: 17 June 2019

\section{References}

1. Kamisawa T, Wood LD, Itoi T, Takaori K. Pancreatic cancer. Lancet. 2016; 388(10039):73-85.

2. Zijlstra M, Bernards N, de Hingh IH, van de Wouw AJ, Goey SH, Jacobs EM, et al. Does long-term survival exist in pancreatic adenocarcinoma? Acta Oncol. 2016;55(3):259-64.

3. Ying H, Dey P, Yao W, Kimmelman AC, Draetta GF, Maitra A, et al. Genetics and biology of pancreatic ductal adenocarcinoma. Genes Dev. 2016;30(4): $355-85$.

4. Kleeff J, Korc M, Apte M, La Vecchia C, Johnson CD, Biankin AV, et al. Pancreatic cancer. Nat Rev Dis Primers. 2016;2:16022.

5. Rahib L, Smith BD, Aizenberg R, Rosenzweig AB, Fleshman JM, Matrisian LM. Projecting cancer incidence and deaths to 2030: the unexpected burden of thyroid, liver, and pancreas cancers in the United States. Cancer Res. 2014; 74(11):2913-21.

6. Labori K, Katz MH, Tzeng CW, Bjornbeth BA, Cvancarova M, Edwin B, et al. Impact of early disease progression and surgical complications on adjuvant chemotherapy completion rates and survival in patients undergoing the surgery first approach for resectable pancreatic ductal adenocarcinoma - a population-based cohort study. Acta Oncol. 2016;55(3):265-77.

7. Neoptolemos JP, Kleeff J, Michl P, Costello E, Greenhalf W, Palmer DH. Therapeutic developments in pancreatic cancer: current and future perspectives. Nat Rev Gastroenterol Hepatol. 2018;15(6):333-48.

8. Burris HA, Moore MJ, Andersen J, Green MR, Rothenberg ML, Modiano MR, et al. Improvements in survival and clinical benefit with gemcitabine as firstline therapy for patients with advanced pancreas cancer: a randomized trial. J Clin Oncol. 1997;15(6):2403-13.

9. Wong A, Soo RA, Yong WP, Innocenti F. Clinical pharmacology and pharmacogenetics of gemcitabine. Drug Metab Rev. 2009;41(2):77-88.

10. Amrutkar M. Gladhaug IP. Pancreatic Cancer Chemoresistance to Gemcitabine. Cancers. 2017;9:157. https://doi.org/10.3390/cancers9110157.

11. Kim MP, Gallick GE. Gemcitabine resistance in pancreatic cancer: picking the key players. Clin Cancer Res. 2008;14(5):1284-5.

12. Neesse A, Michl P, Frese KK, Feig C, Cook N, Jacobetz MA, et al. Stromal biology and therapy in pancreatic cancer. Gut. 2011;60(6):861-8.

13. Kadaba R, Birke H, Wang J, Hooper $S$, Andl CD, Di Maggio F, et al. Imbalance of desmoplastic stromal cell numbers drives aggressive cancer processes. J Pathol. 2013;230(1):107-17

14. Lunardi S, Muschel RJ, Brunner TB. The stromal compartments in pancreatic cancer: are there any therapeutic targets? Cancer Lett. 2014;343(2):147-55.

15. Topalovski M, Brekken RA. Matrix control of pancreatic cancer: new insights into fibronectin signaling. Cancer Lett. 2016;381(1):252-8.

16. Grzesiak JJ, Ho JC, Moossa AR, Bouvet M. The integrin-extracellular matrix axis in pancreatic cancer. Pancreas. 2007;35(4):293-301. 
17. Pothula SP, Xu Z, Goldstein D, Pirola RC, Wilson JS, Apte MV. Key role of pancreatic stellate cells in pancreatic cancer. Cancer Lett. 2016;381(1):194-200.

18. Ferdek PE, Jakubowska MA. Pflugers Arch - Eur J Physiol. 2017:469:1039. https://doi.org/10.1007/s00424-017-1968-0.

19. Apte MV, Wilson JS. Dangerous liaisons: pancreatic stellate cells and pancreatic cancer cells. J Gastroenterol Hepatol. 2012;27(Suppl 2):69-74

20. Liang C, Shi S, Meng Q, Liang D, Ji S, Zhang B, et al. Complex roles of the stroma in the intrinsic resistance to gemcitabine in pancreatic cancer: where we are and where we are going. Exp Mol Med. 2017;49(12):e406.

21. Arumugam $T$, Ramachandran V, Fournier KF, Wang $H$, Marquis L, Abbruzzese $J$, et al. Epithelial to mesenchymal transition contributes to drug resistance in pancreatic cancer. Cancer Res. 2009;69(14):5820-8.

22. Toste PA, Nguyen AH, Kadera BE, Duong M, Wu N, Gawlas I, et al. Chemotherapyinduced inflammatory gene signature and Protumorigenic phenotype in pancreatic CAFs via stress-associated MAPK. Mol Cancer Res. 2016;14(5):437-47.

23. McCarroll JA, Naim S, Sharbeen G, Russia N, Lee J, Kavallaris M, et al. Role of pancreatic stellate cells in chemoresistance in pancreatic cancer. Front Physiol. 2014;5:141

24. Zhang H, Wu H, Guan J, Wang L, Ren X, Shi X, et al. Paracrine SDF-1a signaling mediates the effects of PSCs on GEM chemoresistance through an IL-6 autocrine loop in pancreatic cancer cells. Oncotarget. 2015;6(5):3085-97.

25. Liu $Y$, Li F, Gao F, Xing L, Qin P, Liang $X$, et al. Periostin promotes the chemotherapy resistance to gemcitabine in pancreatic cancer. Tumour Biol. 2016;37(11):15283-91.

26. Zhao X, Fan W, Xu Z, Chen H, He Y, Yang G, et al. Inhibiting tumor necrosis factor-alpha diminishes desmoplasia and inflammation to overcome chemoresistance in pancreatic ductal adenocarcinoma. Oncotarget. 2016; 7(49):81110-22.

27. Dangi-Garimella S, Sahai V, Ebine K, Kumar K, Munshi HG. Three-dimensional collagen I promotes gemcitabine resistance in vitro in pancreatic cancer cells through HMGA2-dependent histone acetyltransferase expression. PLoS One. 2013:8(5):e64566.

28. Miyamoto H, Murakami T, Tsuchida K, Sugino H, Miyake H, Tashiro S. Tumorstroma interaction of human pancreatic cancer: acquired resistance to anticancer drugs and proliferation regulation is dependent on extracellular matrix proteins. Pancreas. 2004;28(1):38-44.

29. Pomianowska E, Sandnes D, Grzyb K, Schjolberg AR, Aasrum M, Tveteraas $\mathbb{H}$, et al. Inhibitory effects of prostaglandin E2 on collagen synthesis and cell proliferation in human stellate cells from pancreatic head adenocarcinoma. BMC Cancer. 2014;14:413.

30. Bachem MG, Schneider E, Gross H, Weidenbach H, Schmid RM, Menke A, et al. Identification, culture, and characterization of pancreatic stellate cells in rats and humans. Gastroenterology. 1998;115(2):421-32.

31. Lenggenhager D, Amrutkar M, Sántha P, Aasrum M, Löhr JM, Gladhaug IP, Verbeke CS. Commonly used pancreatic stellate cell cultures differ phenotypically and in hheir interactions with pancreatic cancer cells. Cells. 2019;8:23. https://doi.org/10.3390/cells8010023.

32. Kanehisa M, Goto S. KEGG: Kyoto encyclopedia of genes and genomes. Nucleic Acids Res. 2000;28(1):27-30.

33. Dennis G Jr, Sherman BT, Hosack DA, Yang J, Gao W, Lane HC, et al. DAVID: database for annotation, visualization, and integrated discovery. Genome Biol. 2003;4(5):P3.

34. Huang d W, Sherman BT, Lempicki RA. Systematic and integrative analysis of large gene lists using DAVID bioinformatics resources. Nat Protoc. 2009; 4(1):44-57.

35. Tjomsland V, Aasrum M, Christoffersen T, Gladhaug IP. Functional heterogeneity in tumor-derived human pancreatic stellate cells: differential expression of HGF and implications for mitogenic signaling and migration in pancreatic cancer cells. Oncotarget. 2017;8(42):71672-84.

36. Pan $X$, Arumugam T, Yamamoto T, Levin PA, Ramachandran V, Ji B, et al. Nuclear factor-kappaB p65/relA silencing induces apoptosis and increases gemcitabine effectiveness in a subset of pancreatic cancer cells. Clin Cancer Res. 2008;14(24):8143-51

37. Fryer RA, Barlett B, Galustian C, Dalgleish AG. Mechanisms underlying gemcitabine resistance in pancreatic cancer and sensitisation by the iMiD lenalidomide. Anticancer Res. 2011:31(11):3747-56.

38. Kim R, Yamauchi T, Husain K, Sebti S, Malafa M. Triciribine phosphate monohydrate, an AKT inhibitor, enhances gemcitabine activity in pancreatic Cancer cells. Anticancer Res. 2015;35(9):4599-604.

39. Hesler RA, Huang JJ, Starr MD, Treboschi VM, Bernanke AG, Nixon AB, et al. TGF- $\beta$-induced stromal CYR61 promotes resistance to gemcitabine in pancreatic ductal adenocarcinoma through downregulation of the nucleoside transporters hENT1 and hCNT3. Carcinogenesis. 2016;37(11): 1041-51.

40. Duxbury MS, Ito H, Zinner MJ, Ashley SW, Whang EE. Inhibition of SRC tyrosine kinase impairs inherent and acquired gemcitabine resistance in human pancreatic adenocarcinoma cells. Clin Cancer Res. 2004;10(7):2307-18.

41. Wilson JS, Pirola RC, Apte MV. Stars and stripes in pancreatic cancer: role of stellate cells and stroma in cancer progression. Front Physiol. 2014;5:52.

42. Masamune A, Watanabe T, Kikuta K, Shimosegawa T. Roles of pancreatic stellate cells in pancreatic inflammation and fibrosis. Clin Gastroenterol Hepatol. 2009;7(11 Suppl):S48-54.

43. Vonlaufen A, Joshi S, Qu C, Phillips PA, Xu Z, Parker NR, et al. Pancreatic stellate cells: partners in crime with pancreatic cancer cells. Cancer Res. 2008;68(7):2085-93.

44. Zhang H, Wu H, Guan J, Wang L, Ren X, Shi X, et al. Paracrine SDF-1alpha signaling mediates the effects of PSCS on GEM chemoresistance through an IL-6 autocrine loop in pancreatic cancer cells. Oncotarget. 2015;6(5):3085-97.

45. Sipos B, Moser S, Kalthoff H, Torok V, Lohr M, Kloppel G. A comprehensive characterization of pancreatic ductal carcinoma cell lines: towards the establishment of an in vitro research platform. Virchows Arch. 2003;442(5):444-52.

46. de Sousa Cavalcante L, Monteiro G. Gemcitabine: metabolism and molecular mechanisms of action, sensitivity and chemoresistance in pancreatic cancer. Eur J Pharmacol. 2014;741:8-16.

47. Achiwa H, Oguri T, Sato S, Maeda H, Niimi T, Ueda R. Determinants of sensitivity and resistance to gemcitabine: the roles of human equilibrative nucleoside transporter 1 and deoxycytidine kinase in non-small cell lung cancer. Cancer Sci. 2004;95(9):753-7.

48. Pankov R, Yamada KM. Fibronectin at a glance. J Cell Sci. 2002;115(Pt 20):3861-3.

49. Erdogan B, Ao M, White LM, Means AL, Brewer BM, Yang L, et al. Cancerassociated fibroblasts promote directional cancer cell migration by aligning fibronectin. J Cell Biol. 2017;216(11):3799-816.

50. Lenselink EA. Role of fibronectin in normal wound healing. Int Wound J. 2015;12(3):313-6.

51. To WS, Midwood KS. Plasma and cellular fibronectin: distinct and independent functions during tissue repair. Fibrogenesis Tissue Repair. 2011;4:21.

52. Labat-Robert J. Fibronectin in malignancy. Semin Cancer Biol. 2002;12(3): 187-95.

53. Crawford DC, Chobanian AV, Brecher P. Angiotensin II induces fibronectin expression associated with cardiac fibrosis in the rat. Circ Res. 1994;74(4): 727-39.

54. Lee JW, Juliano R. Mitogenic signal transduction by integrin- and growth factor receptor-mediated pathways. Mol Cells. 2004;17(2):188-202.

55. Legate KR, Wickstrom SA, Fassler R. Genetic and cell biological analysis of integrin outside-in signaling. Genes Dev. 2009;23(4):397-418.

56. Chadha KS, Khoury T, Yu J, Black JD, Gibbs JF, Kuvshinoff BW, et al. Activated Akt and Erk expression and survival after surgery in pancreatic carcinoma. Ann Surg Oncol. 2006;13(7):933-9.

57. Yamamoto S, Tomita Y, Hoshida Y, Morooka T, Nagano H, Dono K, et al. Prognostic significance of activated Akt expression in pancreatic ductal adenocarcinoma. Clin Cancer Res. 2004;10(8):2846-50.

58. Zheng $C$, Jiao $X$, Jiang $Y$, Sun S. ERK1/2 activity contributes to gemcitabine resistance in pancreatic cancer cells. J Int Med Res. 2013;41(2):300-6.

59. Wang M, Lu X, Dong X, Hao F, Liu Z, Ni G, et al. pERK1/2 silencing sensitizes pancreatic cancer BXPC-3 cell to gemcitabine-induced apoptosis via regulating Bax and BCl-2 expression. World J Surg Oncol. 2015;13:66.

60. Ng SSW, Tsao MS, Chow S, Hedley DW. Inhibition of phosphatidylinositide 3-kinase enhances gemcitabine-induced apoptosis in human pancreatic cancer cells. Cancer Res. 2000;60(19):5451-5.

61. Ng SS, Tsao MS, Nicklee T, Hedley DW. Wortmannin inhibits pkb/akt phosphorylation and promotes gemcitabine antitumor activity in orthotopic human pancreatic cancer xenografts in immunodeficient mice. Clin Cancer Res. 2001;7(10):3269-75.

62. Arlt A, Gehrz A, Muerkoster S, Vorndamm J, Kruse ML, Folsch UR, et al. Role of NF-kappaB and Akt/PI3K in the resistance of pancreatic carcinoma cell lines against gemcitabine-induced cell death. Oncogene. 2003;22(21):3243-51.

63. Sawai $H$, Okada $Y$, Funahashi $H$, Takahashi $H$, Matsuo $Y$, Yasuda $A$, et al. Basement membrane proteins play an important role in the invasive processes of human pancreatic cancer cells. J Surg Res. 2008;144(1):117-23.

64. Shields MA, Dangi-Garimella S, Redig AJ, Munshi HG. Biochemical role of the collagen-rich tumour microenvironment in pancreatic cancer progression. Biochem J. 2012;441(2):541-52. 
65. Januchowski R, Swierczewska M, Sterzynska K, Wojtowicz K, Nowicki M, Zabel $\mathrm{M}$. Increased expression of several collagen genes is associated with drug resistance in ovarian Cancer cell lines. J Cancer. 2016;7(10):1295-310.

66. Iseri OD, Kars MD, Arpaci F, Gunduz U. Gene expression analysis of drugresistant MCF-7 cells: implications for relation to extracellular matrix proteins. Cancer Chemother Pharmacol. 2010;65(3):447-55.

67. Dangi-Garimella S, Krantz SB, Barron MR, Shields MA, Heiferman MJ, Grippo PJ, et al. Three-dimensional collagen I promotes gemcitabine resistance in pancreatic cancer through MT1-MMP-mediated expression of HMGA2. Cancer Res. 2011;71(3):1019-28.

68. Ruess DA, Gorgulu K, Wormann SM, Algul H. Pharmacotherapeutic Management of Pancreatic Ductal Adenocarcinoma: current and emerging concepts. Drugs Aging. 2017;34(5):331-57.

69. Whatcott CJ, Han H, Von Hoff DD. Orchestrating the tumor microenvironment to improve survival for patients with pancreatic Cancer: normalization, not destruction. Cancer J. 2015;21(4):299-306.

\section{Publisher's Note}

Springer Nature remains neutral with regard to jurisdictional claims in published maps and institutional affiliations.

Ready to submit your research? Choose BMC and benefit from:

- fast, convenient online submission

- thorough peer review by experienced researchers in your field

- rapid publication on acceptance

- support for research data, including large and complex data types

- gold Open Access which fosters wider collaboration and increased citations

- maximum visibility for your research: over $100 \mathrm{M}$ website views per year

At BMC, research is always in progress.

Learn more biomedcentral.com/submissions 